\title{
Does Early ART Initiation is Better over Later ART Initiation in TB/HIV Co-infected Patients? A Retrospective Cohort Study;
}

\section{CURRENT STATUS: UNDER REVIEW}

AIDS Research and Therapy $\triangle B M C$

Desilu Mahari Desta

Mekelle University College of Health Sciences

v desiluma45@gmail.comCorresponding Author

ORCiD: https://orcid.org/0000-0003-0332-9517

Tesfay Mehari Atey

Fikermaryam Girma

Kald Beshir Tuem

Abadi Kahsu Gebre

Hagos Gidey

Mola Kahssay

Hiluf Hindeya Gebreyesus

Solomon Weldegebreal Asgedom

DOI:

10.21203/rs.3.rs-15492/v1

SUBJECT AREAS

Infectious Diseases

KEYWORDS

Treatment outcome, early ART initiation, late ART initiation, TB/HIV co-infection 
Abstract

Background: The co-infection of TB/HIV poses a significant burden in the health care system of developing countries like Ethiopia. There are conflicting results on preference of the time to initiate anti-retroviral therapy (ART) and hence assessing the survival experience and treatment outcomes associated with ART initiation is crucial to settle the controversies. The study compared the treatment outcomes in early versus later ART initiation in TB/HIV co-infected patients.

Methods: A retrospective cohort study was conducted in Ayder Comprehensive Specialized Hospital and Mekelle Referral Hospital on 77 and 105 patients that started ART early and lately, respectively. An assumption for proportional hazard was met. Kaplan-Meier and life-table analyses were used to compare survival curves; and an independent samples t -test was used to compare means of the continuous variables between the two cohorts. Moreover, incidence per 100 persons-years were employed to crudely determine new morality rates and Cox regression analysis was done to find out the effects of independent variables on the outcome variables.

Results: The mean survival time was 5.8 months after ART initiation. A 9.9 and 5.5 new incident mortality rates per 10,000 persons-years for the early and late ART initiation were observed, respectively. There was a statistically significant difference in mean CD4 $+\mathrm{T}$ cells between early $(208.20 \pm 11.94$ cells $/ \mathrm{mm} 3)$ and late $(245.94 \pm 11.69$ cells $/ \mathrm{mm} 3)$ ART initiators (t $180=-2.213, p$ $<0.028$ ). Additionally, late initiators had a better survival chance at all levels of time (Log Rank c 2 $=5.56, \mathrm{p}<0.018$ ) than early initiators. Having normal body mass index [adjusted hazard ratio $[A H R=0.263 ; 95 \%$ confidence interval $[\mathrm{Cl}]: 0.089-0.778]$ and having a 'working' baseline functional status $[A H R=0.151 ; 95 \% \mathrm{Cl}: 0.054-0.427]$ were found to be preventive factors from death. However, patients with $<250 \mathrm{CD} 4+\mathrm{T}$ cells/mm 3 were more likely to die earlier [AHR=12.023; 95\%: 1.58891.005] than their counterpart groups.

Conclusion: This study highlights that TB/HIV co-infected patients with moderate immunosuppression who started their ART early had worse outcome than those who started their ART lately. Moreover, body mass index, baseline functional status, and CD4 count were found to be independent predictors of mortality. 
Keywords: Treatment outcome, early ART initiation, late ART initiation, TB/HIV co-infection

Full Text

Due to technical limitations, full-text HTML conversion of this manuscript could not be completed.

However, the manuscript can be downloaded and accessed as a PDF.

Figures

Total number of TB/HIV co-infected patients $[\sim \mathrm{n}=2245]$

Total number of patietns with missing medical records, taking ART before the index year and with unknown TB and ART initaition time $[\sim \mathrm{n}=901]$

Total number of TB/HIV coinfected patients not taking ART $[\sim \mathrm{n}=23]$

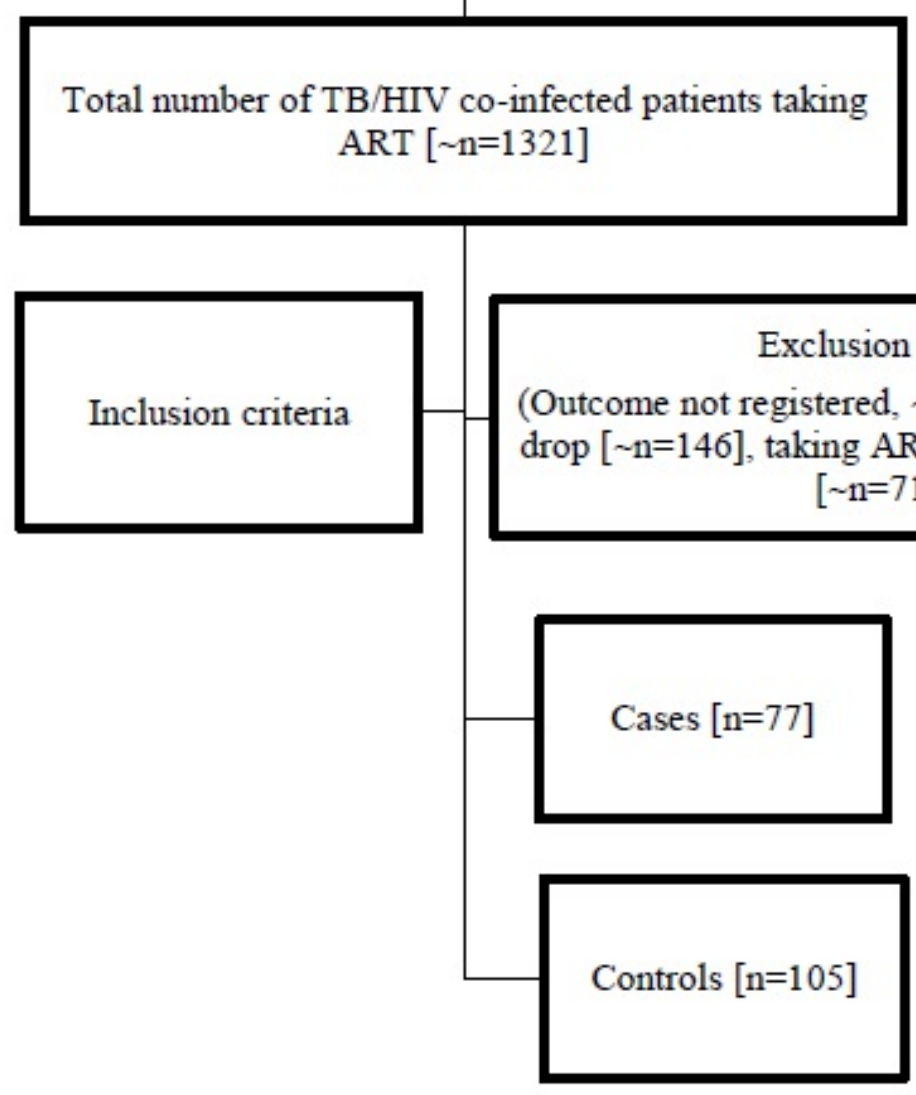

Figure 1

A diagram that shows eligible patients' selection process. 


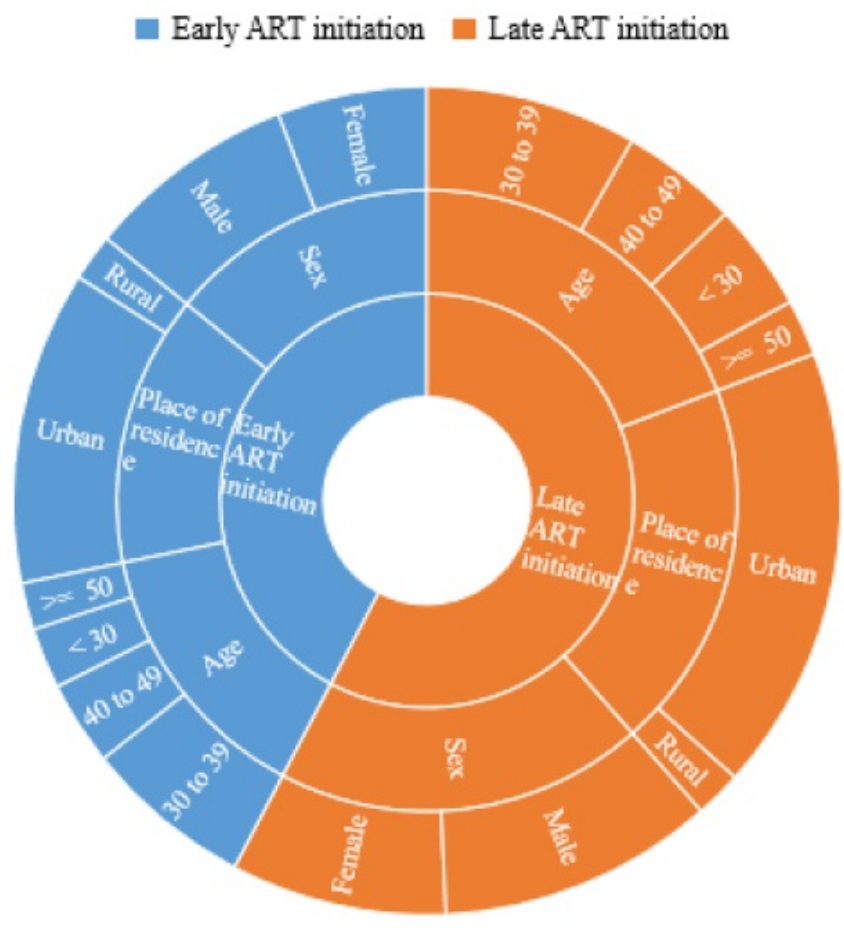

Figure 2

Socio-demographic characteristics of TB/HIV co-infected patients in two governmental hospitals of Mekelle, Ethiopia, 2012-2017. 


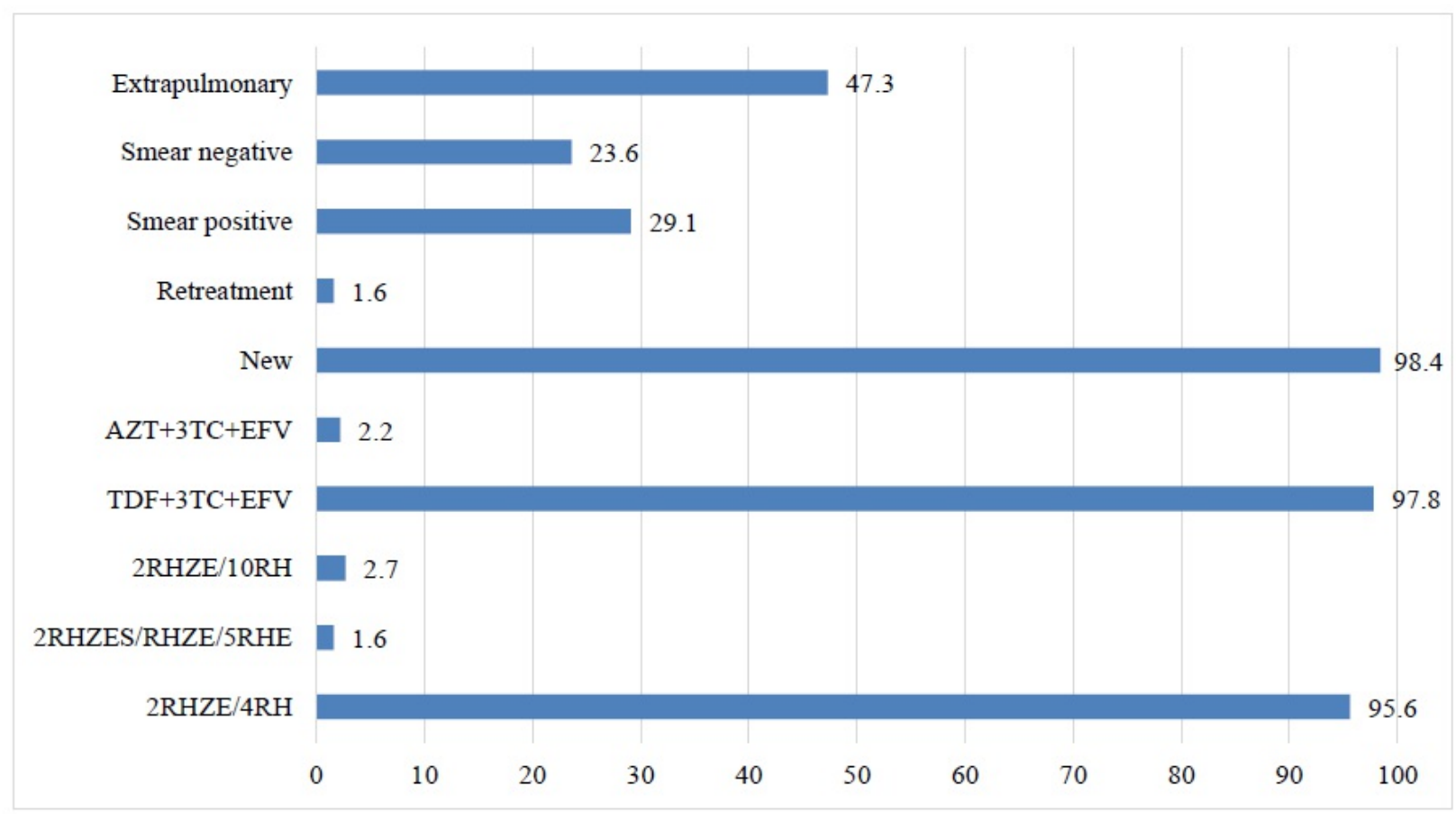

3TC=Lamivudine; AZT=Zidovudine; E=Ethambutol; EFV=Efavirenz; $\mathrm{H}=$ =Isoniazid;

$\mathrm{R}=$ Rifampin; $\mathrm{S}=$ Streptomycin; TDF=Tenofovir; $\mathrm{Z}=$ Pyrazinamide

Figure 3

Status and classification of TB at diagnosis stage, type of ART and TB treatment regimen among TB/HIV co-infected patients in two governmental hospitals of Mekelle, Ethiopia, 2012-2017. 


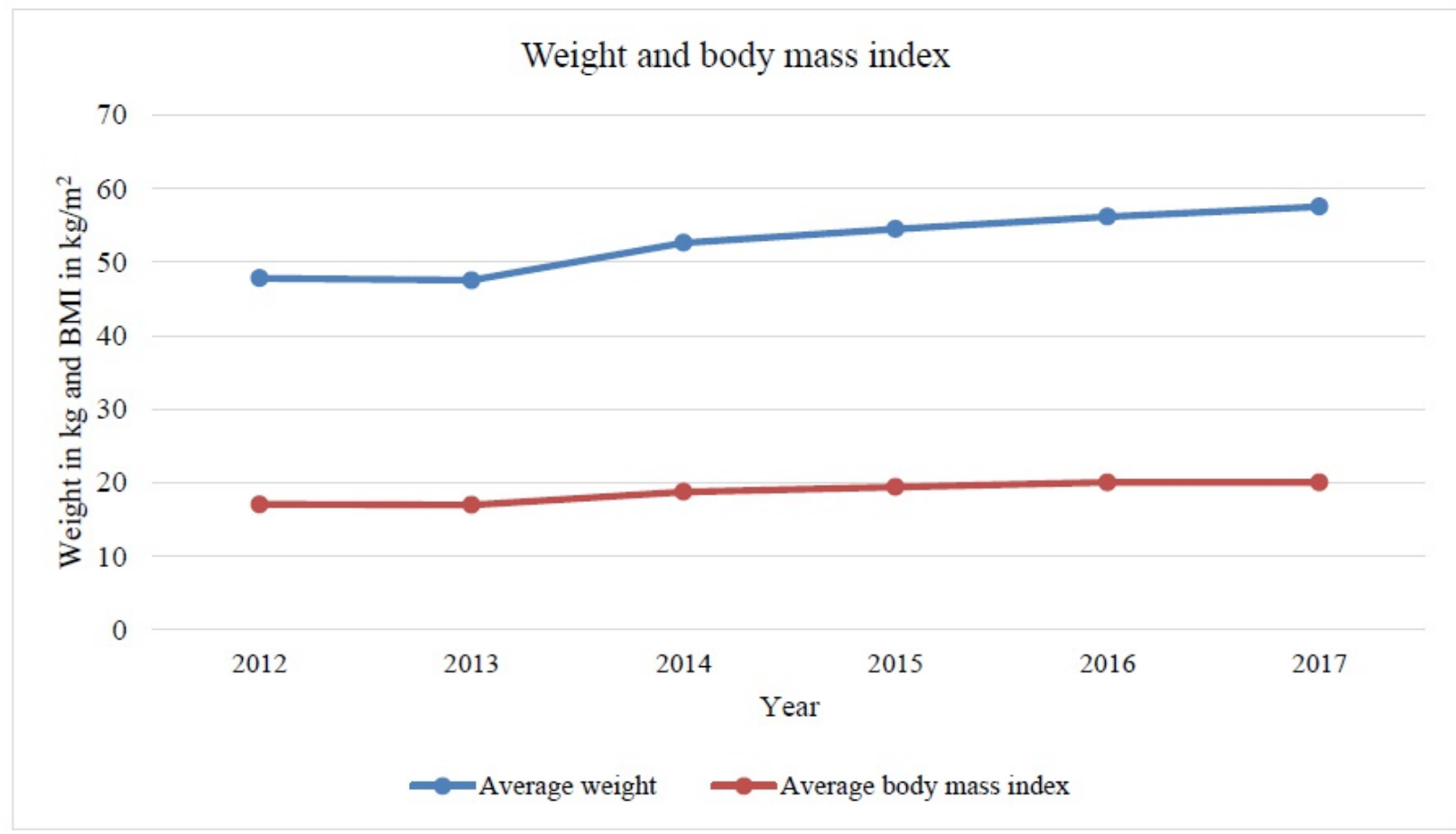

Figure 4

Trend of weight and body mass index among TB/HIV co-infected patients in two governmental hospitals of Mekelle, Ethiopia, 2012-2017. 


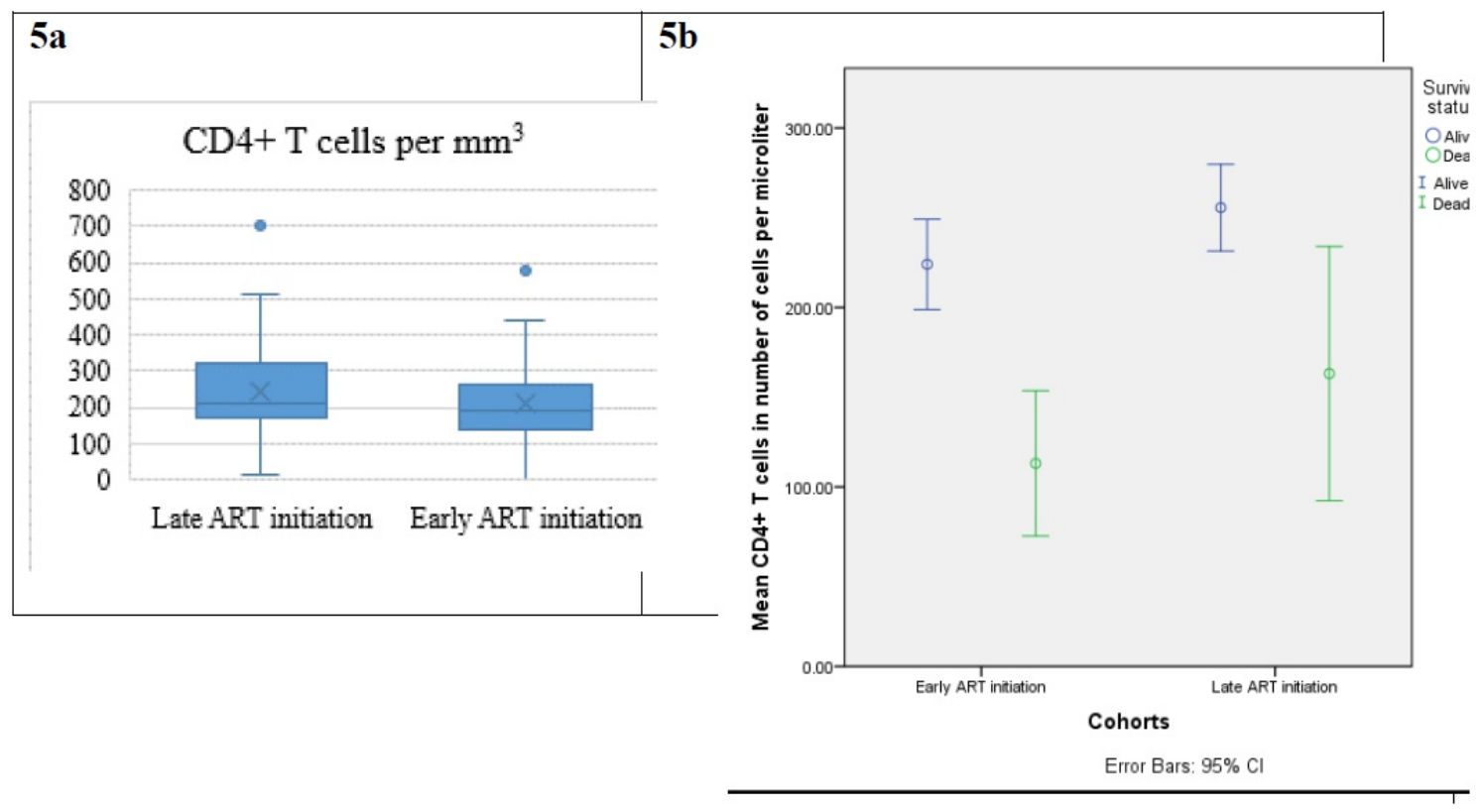

Figure 5

Mean CD4+ T cells for early and late ART initiation (5a), disaggregated by survival status

(5b), among TB/HIV co-infected patients in two governmental hospitals of Mekelle, Ethiopia, 2012-2017. 


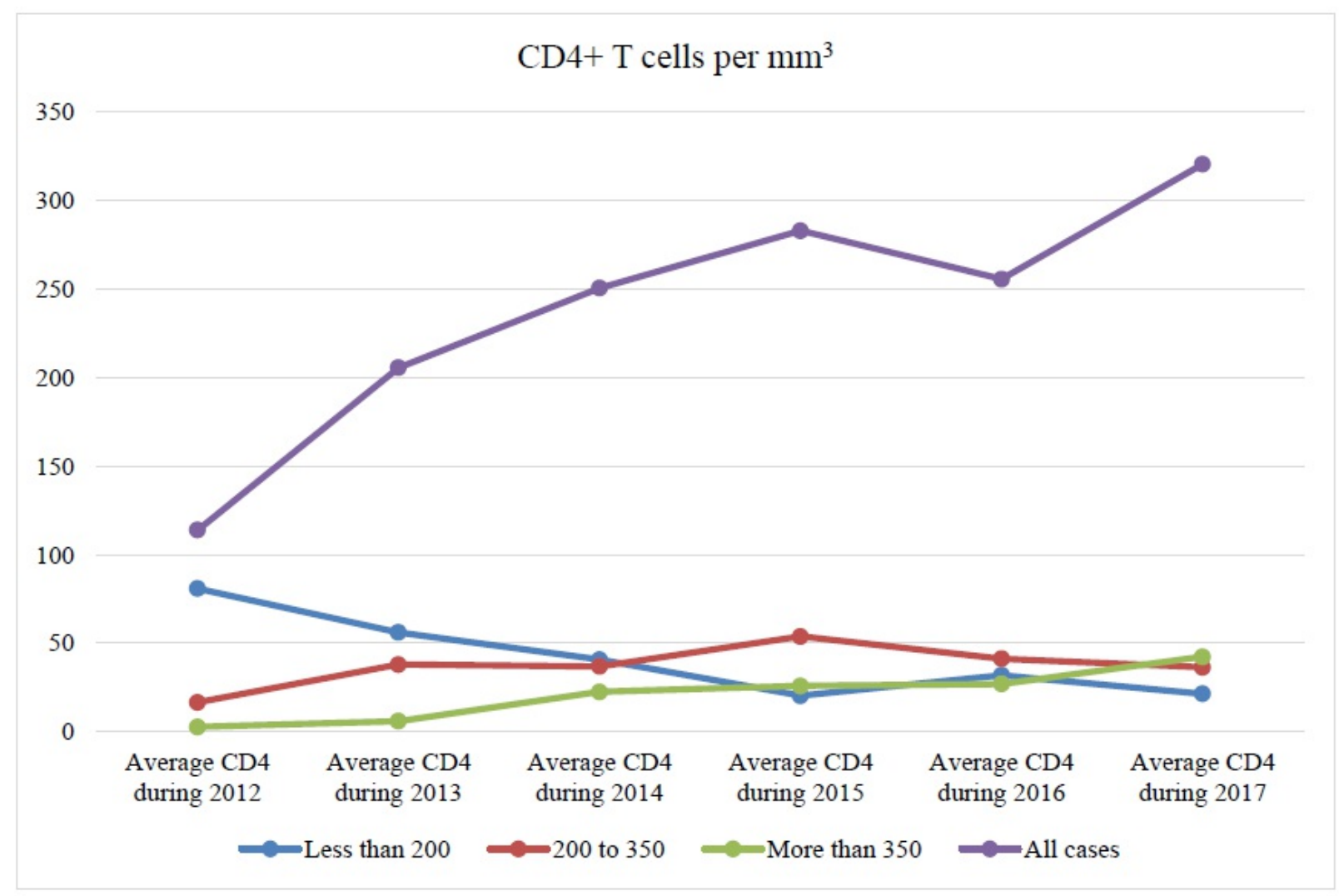

Figure 6

Trend of CD4+ T cells per millimeter cubic among TB/HIV co-infected patients in two governmental hospitals of Mekelle, Ethiopia, 2012-2017. 


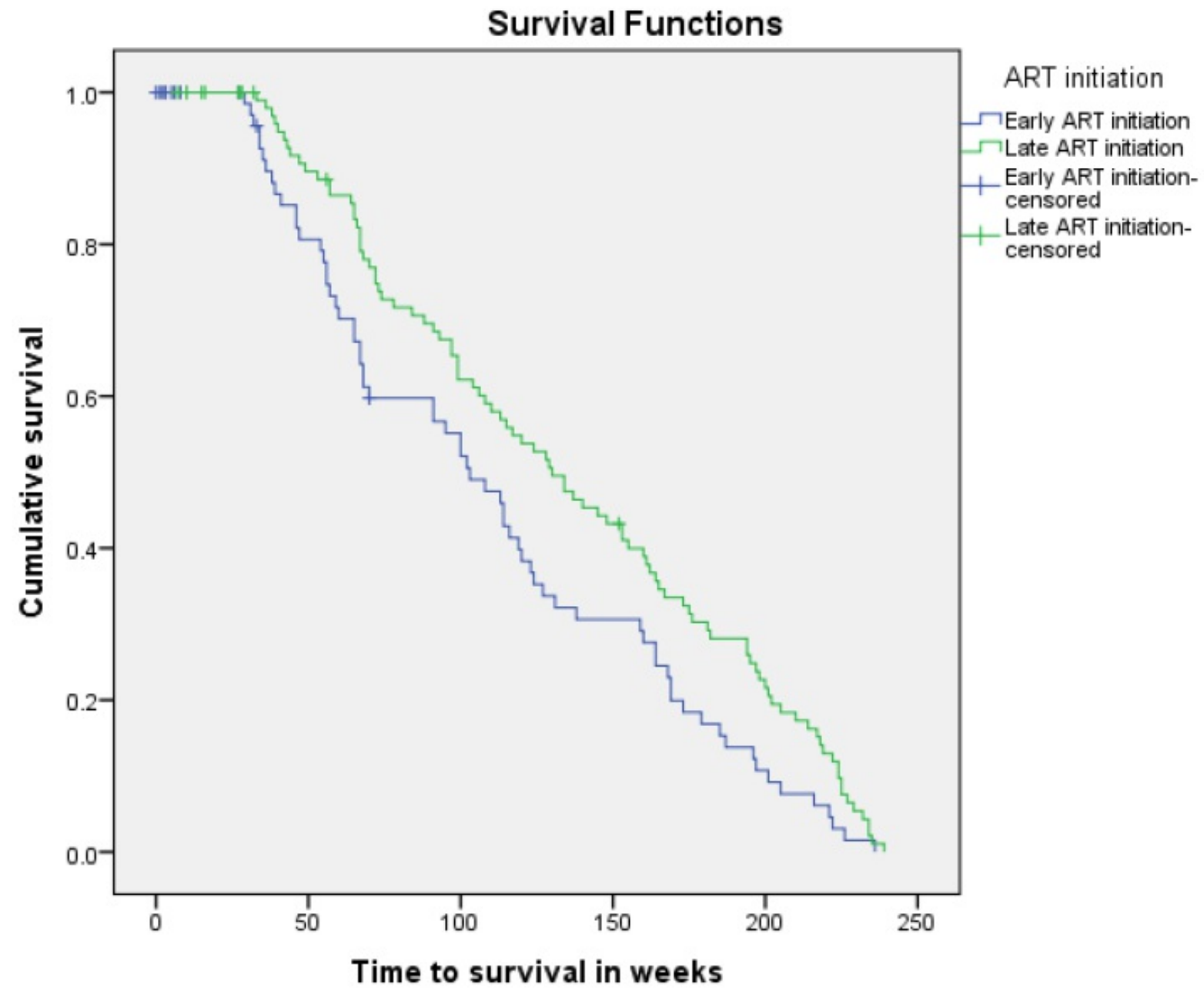

Figure 7

Comparison of survival status of early and late ART initiation among TB/HIV co-infected patients in two governmental hospitals of Mekelle, Ethiopia, 2012-2017. 